\title{
Mucolipidosis type II
}

INSERM

\section{Source}

INSERM. (1999). Orphanet: an online rare disease and orphan drug data base.

Mucolipidosis type II. ORPHA:576

Mucolipidosis II (MLII) is a slowly progressive lysosomal disorder characterized by growth retardation, skeletal abnormalities, facial dysmorphism, stiff skin, developmental delay and cardiomegaly. 